\title{
Reduction of Decentralized Control Problems to Tractable Representations
}

\author{
Laurent Lessard ${ }^{1}$ \\ Sanjay Lall ${ }^{2}$ \\ IEEE Conference on Decision and Control, pp. 1621-1626, 2009
}

\begin{abstract}
For decentralized control problems with quadratically invariant information constraints, the optimal controller may be found efficiently. In this paper, we show that there are systems which are not quadratically invariant but reduce to systems that are. We call the requisite property internal quadratic invariance. We present an associated reduction procedure, and illustrate our method with examples.
\end{abstract}

\section{Introduction}

In decentralized control problems, each controller has access to some subset of the measurements and must control some subset of the actuators. Such situations are of practical interest because it is often infeasible to have a single computer process all the information and make all the decisions. For example, we may be trying to design an auto-pilot for a swarm of vehicles flying in formation, where each vehicle only has access to noisy local measurements of the positions of its nearest neighbors. Another example is packet routing in networks. Each switch must make decisions based on local information, but the goal is to optimize the efficiency of the whole network.

Many decentralized control problems are computationally intractable. However, if a problem has a quadratically invariant (QI) information structure [8, 9], then optimal controllers may be efficiently computed. In this paper, we expand the QI class by showing that some nonQI problems can be reduced to QI problems and thereby solved. We call such problems internally quadratically invariant. We also provide a sufficient condition for when such a reduction exists, and a partial converse.

The paper is organized as follows. In Section 2, we review some fundamental concepts including quadratic invariance, and in Section 3, we define internal quadratic invariance and equivalence of systems. In Section 4 we present our main results, and in Section 5 we show two illustrative examples.

\footnotetext{
${ }^{1}$ L. Lessard is with the Department of Aeronautics and Astronautics at Stanford University, Stanford, CA 94305, USA. lessard@stanford. edu

${ }^{2} \mathrm{~S}$. Lall is with the Department of Electrical Engineering and Aeronautics and Astronautics at Stanford University, Stanford, CA 94305, USA. lall@stanford.edu

${ }^{3}$ This work was supported by NSF grant number 0642839
}

Prior Work. When the system to be controlled has a linear plant, quadratic cost, and Gaussian noise (LQG), the optimal centralized controller is linear, and can be computed efficiently. However, in 1968, Witsenhausen [10] provided a now famous counter-example showing that for decentralized control, the optimal LQG controller is not linear in general. Subsequently, Blondel and Tsitsiklis [2] proved that a certain class of decentralized control problems is NP-hard.

This led to an effort to characterize which decentralized problems have optimal controllers that are linear. Radner [6] showed that this was true for a special class called static team decision problems. Ho and Chu [4] generalized Radner's result by showing that the larger class of partially nested systems could be converted into static team decision problems and hence solved easily.

Optimal decentralized control problems with quadratically invariant information constraints were shown to be tractable in $[7,8,9]$. Computational tractability and linearity of the optimal controller arise because in these cases the set of achievable closed-loop maps is convex.

The QI class is extremely wide, but does not cover all tractable decentralized control problems, nor all the problems for which the optimal controller is linear. For example, Bansal and Basar [1] showed that by using a different quadratic cost function in the Witsenhausen counter-example, the problem is still not QI, but has a linear optimal solution. In section 5 , we present two examples of systems that fail to be QI, but are still tractable and have linear optimal solutions.

\section{Preliminaries}

We review some properties of matrices of rational functions, see for example $[5, \S 6.3, \S 6.5]$, and the notion of quadratic invariance $[8,9]$. We also show an illustrative example.

Define $\mathcal{R}^{m \times n}$ to be the set of $m \times n$ matrices in which each entry is a real-rational function. In addition, let $\mathcal{R}_{p}^{m \times n}$ denote $m \times n$ matrices in which each entry is a proper real-rational function, and let $\mathcal{R}_{s p}^{m \times n}$ denote $m \times n$ matrices in which each entry is a strictly proper realrational function. We omit the superscript when the dimensions are to be inferred by context.

We state the following lemma without proof. For an introduction to normal rank and related concepts, see [5]. 
Lemma 1. Suppose $A \in \mathcal{R}^{m \times n}$, and $B \in \mathcal{R}^{n \times k}$.

i) $\operatorname{nrank} A B \leq \min \{\operatorname{nrank} A, \operatorname{nrank} B\}$.

ii) If $A$ is tall and has full normal rank, $\operatorname{nrank} A B=\operatorname{nrank} B$

We say that $A$ is invertible if it is square and its determinant $\operatorname{det} A(s)$ is not identically zero. Note that we do not require $A$ or its inverse to be proper. The following result states that, regardless of whether $A$ has full normal rank or not, we can factor it as the product of two matrices with full normal rank. This is constructed by writing $A$ in Smith-McMillan form. See [5, §6.5.2].

Lemma 2. Suppose $A \in \mathcal{R}^{m \times n}$. There exists a factorization $A=U_{1} U_{2}$, where $U_{1} \in \mathcal{R}^{m \times r}$ is tall and full normal rank, $U_{2} \in \mathcal{R}^{r \times n}$ is wide and full normal rank, and $r=\operatorname{nrank} A$.

The plant $P \in \mathcal{R}^{m \times n}$ is partitioned as

$$
\left[\begin{array}{l}
z \\
y
\end{array}\right]=\left[\begin{array}{ll}
P_{11} & P_{12} \\
P_{21} & P_{22}
\end{array}\right]\left[\begin{array}{l}
w \\
u
\end{array}\right]
$$

with $m=m_{1}+m_{2}$ and $n=n_{1}+n_{2}$, and $P_{11} \in \mathcal{R}^{m_{1} \times n_{1}}$, $P_{12} \in \mathcal{R}^{m_{1} \times n_{2}}, P_{21} \in \mathcal{R}^{m_{2} \times n_{1}}$, and $P_{22} \in \mathcal{R}^{m_{2} \times n_{2}}$. The controller $K \in \mathcal{R}^{n_{2} \times m_{2}}$ is connected via $u=K y$, and these equations are illustrated in Figure 1.

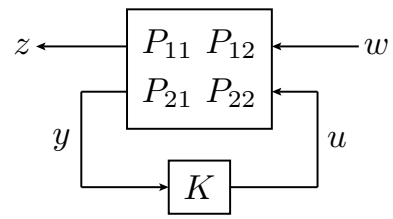

Figure 1: Closed-loop interconnection

When $\left(I-P_{22} K\right)$ is invertible, we say the interconnection is well-posed, and the closed-loop map is

$$
f(P, K)=P_{11}+P_{12} K\left(I-P_{22} K\right)^{-1} P_{21} .
$$

In this paper, we will consider sets of interconnections parametrized by $K \in S$. We call the subspace $S \subset$ $\mathcal{R}^{n_{2} \times m_{2}}$ the information constraint. We will often refer to the pair $(P, S)$ when we want to consider all the well-posed interconnections between $P$ and a controller belonging to $S$. The set of achievable closed-loop maps is denoted as

$$
f(P, S)=\left\{f(P, K) \mid K \in S,\left(I-P_{22} K\right) \text { is invertible }\right\} .
$$

For $U \in \mathcal{R}^{m_{2} \times p}$, define $S U=\{K U \mid K \in S\}$ to be the transformed information constraint. If $V \in \mathcal{R}^{q \times n_{2}}$, we similarly define the sets $V S$ and $V S U$. We now turn to quadratic invariance of such constraints.

Definition 3. $(P, S)$ is quadratically invariant ( $Q I)$, if for all $K \in S$, we have $K P_{22} K \in S$.
Under some additional technical conditions, quadratic invariance is necessary and sufficient for the information constraint $S$ to be preserved under feedback. This allows us to formulate the constrained controller synthesis problem as a convex optimization problem. The following definition and theorem are from [8].

Definition 4. If $S \subset \mathcal{R}_{p}^{n_{2} \times m_{2}}$ is a subspace, we say $S$ is frequency aligned if there exists a subspace $S_{0} \subset$ $\mathbb{C}^{n_{2} \times m_{2}}$ such that

$$
S=\left\{K \in \mathcal{R}_{p}^{n_{2} \times m_{2}} \mid K(j \omega) \in S_{0} \text { for almost all } \omega \in \mathbb{R}\right\}
$$

Theorem 5. Suppose $P_{22} \in \mathcal{R}_{s p}^{m_{2} \times n_{2}}, S \subset \mathcal{R}_{p}^{n_{2} \times m_{2}}$ is a frequency aligned subspace, and $(P, S)$ is $Q I$. Then $K$ is optimal for the problem

$$
\begin{array}{ll}
\text { minimize } & \|f(P, K)\| \\
\text { subject to } & K \in S
\end{array}
$$

if and only if $K=-Q\left(I-P_{22} Q\right)^{-1}$ and $Q$ is optimal for

$$
\begin{array}{ll}
\text { minimize } & \left\|P_{11}-P_{12} Q P_{21}\right\| \\
\text { subject to } & Q \in S
\end{array}
$$

Equation (2) is a convex optimization problem and can be easily solved in most cases; see for example [3]. In the sections that follow, we will show that certain nonQI problems can be transformed into QI problems and solved using a result such as Theorem 5 . The results in this paper hold for plants and controllers that are general rational functions. We do not enforce stability or properness constraints.

\section{Internal Quadratic Invariance}

We say that $(P, S)$ and $(\tilde{P}, \tilde{S})$ are equivalent if they have the same sets of possible closed-loop maps. That is, $f(P, S)=f(\tilde{P}, \tilde{S})$. Note that if $(\tilde{P}, \tilde{S})$ is not QI, but is equivalent to $(P, S)$, which is QI, then we may solve the corresponding optimization problem using a result such as Theorem 5 . Then, equivalence implies that $(\tilde{P}, \tilde{S})$ has the same optimal value. This motivates the following new definition.

Definition 6. $(\tilde{P}, \tilde{S})$ is internally quadratically invariant (internally QI) if there exists a quadratically invariant system $(P, S)$ that is equivalent to $(\tilde{P}, \tilde{S})$.

We now show an example of an internally QI system.

$$
\begin{aligned}
& \tilde{P}=\left[\begin{array}{c:ccc}
P_{11} & 2 B_{1} & 3 B_{2} & 5 B_{2} \\
\hdashline \frac{1}{2} C_{1} & G_{1} & 0 & 0 \\
\frac{1}{3} C_{1} & \frac{2}{3} G_{1} & 0 & 0 \\
\frac{1}{5} C_{2} & \frac{2}{5} G_{2} & \frac{3}{5} G_{3} & G_{3}
\end{array}\right] \\
& \tilde{S}=\left\{\left[\begin{array}{ccc}
K_{1} & 0 & 0 \\
0 & K_{2} & 0 \\
0 & 0 & K_{3}
\end{array}\right] \mid K_{i} \in \mathcal{R}\right\}
\end{aligned}
$$


Here $P_{11}, B_{i}, C_{i}, G_{i}$, and $K_{i}$ are matrices of rational functions of compatible size. The matrix $\tilde{P}$ is partitioned into its four blocks as shown; this does not denote a statespace representation. The information constraint $\tilde{S}$ is the subspace of controllers with a block-diagonal structure. Note that $\tilde{K} \tilde{P}_{22} \tilde{K}$ is not block diagonal for all block diagonal $\tilde{K}$, so $(\tilde{P}, \tilde{S})$ is not QI. Now consider the system

$$
P=\left[\begin{array}{c:cc}
P_{11} & B_{1} & B_{2} \\
\hdashline C_{1} & G_{1} & 0 \\
C_{2} & G_{2} & G_{3}
\end{array}\right] \quad S=\left\{\left[\begin{array}{cc}
K_{1} & 0 \\
K_{2} & K_{3}
\end{array}\right] \mid K_{i} \in \mathcal{R}\right\} .
$$

Here, the information constraint $S$ is the subspace of block lower-triangular controllers. This new pair is QI, since $K P_{22} K$ is a product of block lower-triangular matrices, and hence is block lower-triangular itself.

One can show that these two systems are equivalent by computing both closed-loop maps and verifying that they are the same for all choices of $K_{i}$. In other words, $(\tilde{P}, \tilde{S})$ is internally QI. We will analyze this example in more detail in Section 5.

\section{Reduction}

In this section, we show two ways of generating equivalent systems: output reduction and input reduction. Both preserve quadratic invariance.

\subsection{Output Reduction}

We first consider output reduction, illustrated in Figure 2. We say $(P, S)$ is an output transformation of $(\tilde{P}, \tilde{S})$ under $U \in \mathcal{R}$ if

$$
\left[\begin{array}{ll}
\tilde{P}_{11} & \tilde{P}_{12} \\
\tilde{P}_{21} & \tilde{P}_{22}
\end{array}\right]=\left[\begin{array}{cc}
I & 0 \\
0 & U
\end{array}\right]\left[\begin{array}{ll}
P_{11} & P_{12} \\
P_{21} & P_{22}
\end{array}\right] \text { and } S=\tilde{S} U
$$
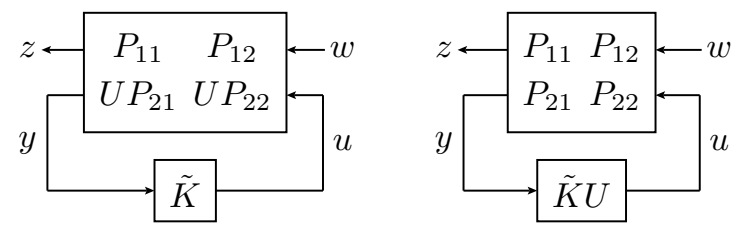

Figure 2: Output transformation.

Lemma 7. Systems related by an output transformation are equivalent. That is, suppose $(P, S)$ is an output transformation of $(\tilde{P}, \tilde{S})$ under $U$. Then we have $f(\tilde{P}, \tilde{S})=f(P, S)$.

Proof. It is straightforward to verify that for all $\tilde{K}$ such that the closed-loop interconnection $(\tilde{P}, \tilde{K})$ is well-posed, $f(\tilde{P}, \tilde{K})=f(P, \tilde{K} U)$. Equivalence follows directly.

Next, we show that output transformations preserve quadratic invariance.
Theorem 8. Suppose $(P, S)$ is an output transformation of $(\tilde{P}, \tilde{S})$ under $U$.

i) If $(\tilde{P}, \tilde{S})$ is $Q I$, then $(P, S)$ is $Q I$.

ii) If $U$ is wide and has full normal rank, then the converse holds: If $(P, S)$ is $Q I$, then $(\tilde{P}, \tilde{S})$ is $Q I$.

iii) If $(P, S)$ is $Q I$, then $(\tilde{P}, \tilde{S})$ is internally $Q I$.

Proof. Suppose $(\tilde{P}, \tilde{S})$ is QI. By definition,

$$
\tilde{K} \in \tilde{S} \Longrightarrow \tilde{K} U P_{22} \tilde{K} \in \tilde{S} .
$$

Choose any $K \in S$. We will show that $K P_{22} K \in S$ and therefore $(P, S)$ is QI. By the definition of the output transformation, there exists some $\tilde{K} \in \tilde{S}$ such that $K=\tilde{K} U$. From (3), $\tilde{K} U P_{22} \tilde{K} \in \tilde{S}$. It follows that $\tilde{K} U P_{22} \tilde{K} U \in S$, and hence $K P_{22} K \in S$. This completes the proof of item i.

Suppose conversely that $(P, S)$ is QI. By definition, for every $K \in S$ we have $K P_{22} K \in S$. Choose any $\tilde{K} \in \tilde{S}$. We will show that if $U$ is wide and has full normal rank, $\tilde{K} U P_{22} \tilde{K} \in \tilde{S}$. By the definition of the output transformation, $\tilde{K} U \in S$, and so $\tilde{K} U P_{22} \tilde{K} U \in S$. Since $S=\tilde{S} U$, there must exist some $\tilde{H} \in \tilde{S}$ such that:

$$
\tilde{H} U=\tilde{K} U P_{22} \tilde{K} U
$$

If we further suppose that $U$ is wide and has full normal rank, $U$ is right-invertible. We conclude from (4) that $\tilde{H}=\tilde{K} U P_{22} \tilde{K} \in \tilde{S}$. This completes the proof of item ii. The final item follows immediately from the definition of internal quadratic invariance and Lemma 7.

Theorem 8 implies that if we have a pair $(\tilde{P}, \tilde{S})$, that is not QI, and we can find $U$ that factors $\tilde{P}$, then the equivalent system $(P, S)$ might be QI. Our next step is to characterize all the output transformations that have the potential to make the system QI. By Lemmma 2, we may restrict our attention without loss of generality to output transformations where $U$ is strictly tall and has full normal rank. Note that all such transformations reduce the number of outputs $m_{2}$ of the system. An output transformation with a tall and full normal rank $U$ is called an output reduction. If $U$ is strictly tall, we call it a strict output reduction.

Definition 9. We say $(P, S)$ is output-minimal if the matrix $\left[\begin{array}{ll}P_{21} & P_{22}\end{array}\right]$ is wide and has full normal rank.

Theorem 10. If $(P, S)$ and $\left(P^{\prime}, S^{\prime}\right)$ are output-minimal output reductions of $(\tilde{P}, \tilde{S})$ under $U_{1}$ and $U_{2}$ respectively, then $m_{2}=m_{2}^{\prime}$, and $(P, S)$ is $Q I$ if and only if $\left(P^{\prime}, S^{\prime}\right)$ is $Q I$.

Proof. By assumption, there exist tall and full normal rank $U_{1}$ and $U_{2}$ such that:

$$
\begin{gathered}
{\left[\begin{array}{ll}
\tilde{P}_{21} & \tilde{P}_{22}
\end{array}\right]=U_{1}\left[\begin{array}{ll}
P_{21} & P_{22}
\end{array}\right]=U_{2}\left[\begin{array}{ll}
P_{21}^{\prime} & P_{22}^{\prime}
\end{array}\right],} \\
\tilde{K} U_{1}=K \text { and } \tilde{K} U_{2}=K^{\prime} \text { for all } \tilde{K} \in \tilde{S}
\end{gathered}
$$


where $\left[\begin{array}{ll}P_{21} & P_{22}\end{array}\right]$ and $\left[\begin{array}{ll}P_{21}^{\prime} & P_{22}^{\prime}\end{array}\right]$ are wide and have full normal rank. Applying Lemma 1 to (5), we find that $U_{1}$ and $U_{2}$ must have the same normal rank, and hence the same size. Thus $m_{2}=m_{2}^{\prime}$. Tall matrices with full normal rank are left-invertible, so

$$
\left[\begin{array}{ll}
P_{21} & P_{22}
\end{array}\right]=\left(U_{1}^{\dagger} U_{2}\right)\left[\begin{array}{ll}
P_{21}^{\prime} & P_{22}^{\prime}
\end{array}\right],
$$

where $A^{\dagger}$ is Moore-Penrose pseudoinverse for full-rank matrices: $\left(A^{T} A\right)^{-1} A^{T}$ when $A$ is tall and $A^{T}\left(A A^{T}\right)^{-1}$ when $A$ is wide. It is straightforward to verify that

$$
K\left(U_{1}^{\dagger} U_{2}\right)=K^{\prime}
$$

Equations (7) and (8) imply that $\left(P^{\prime}, S^{\prime}\right)$ is an output transformation of $(P, S)$ under $U_{1}^{\dagger} U_{2}$. Similarly, one can show that $(P, S)$ is an output transformation of $\left(P^{\prime}, S^{\prime}\right)$ under $U_{2}^{\dagger} U_{1}$. Applying Theorem 8, we conclude that $(P, S)$ is QI if and only if $\left(P^{\prime}, S^{\prime}\right)$ is QI. Indeed, these output transformations are inverses of one another: $\left(U_{1}^{\dagger} U_{2}\right)^{-1}=U_{2}^{\dagger} U_{1}$.

Lemma 11. A strict output reduction is not possible on an output-minimal system.

Proof. This justifies our use of the term "minimality". If we could reduce $\tilde{m}_{2}$ to some $m_{2}<\tilde{m}_{2}$ by using an output reduction, there would exist a tall and full normal rank $U$ such that:

$$
\left[\begin{array}{ll}
\tilde{P}_{21} & \tilde{P}_{22}
\end{array}\right]=U\left[\begin{array}{ll}
P_{21} & P_{22}
\end{array}\right]
$$

but then $\tilde{m}_{2}=\operatorname{nrank}\left[\begin{array}{cc}\tilde{P}_{21} & \tilde{P}_{22}\end{array}\right] \leq \operatorname{nrank} U=m_{2}$, which is a contradiction.

Theorem 12. There always exists an output reduction to an output-minimal system.

Proof. Consider the pair $(\tilde{P}, \tilde{S})$. From Lemma 2, we can factor:

$$
\left[\begin{array}{ll}
\tilde{P}_{21} & \tilde{P}_{22}
\end{array}\right]=U\left[\begin{array}{ll}
P_{21} & P_{22}
\end{array}\right],
$$

where $U$ is $m_{2} \times r$ and has full normal rank, and $\left[\begin{array}{ll}P_{21} & P_{22}\end{array}\right]$ is $r \times n$ and has full normal rank. Applying Lemma 1 , we have $r=\operatorname{nrank}\left[\begin{array}{ll}\tilde{P}_{21} & \tilde{P}_{22}\end{array}\right]=$ nrank $\left[\begin{array}{ll}P_{21} & P_{22}\end{array}\right]$ and so we conclude that $\left[\begin{array}{ll}P_{21} & P_{22}\end{array}\right]$ has full normal rank and thus is output-minimal.

Corollary 13. Consider $(\tilde{P}, \tilde{S})$, and consider the set of all possible $(P, S)$ that can be obtained from it via an output transformation. This set contains a QI system if and only if an output-minimal reduction of $(\tilde{P}, \tilde{S})$ is $Q I$.

Proof. This follows from Theorems 8, 10, and 12.

Corollary 13 provides a sufficient condition for internal quadratic invariance. It also provides a partial converse in the following sense: if we test the output-minimal system constructed using Theorem 12 and the result is not QI, then no output transformation can yield a QI system.

\subsection{Input Reduction}

The notion of input reduction is analogous to that of output reduction, and results can be derived which parallel those from section 4.1. We only include some of them here and omit the proofs.

We say $(P, S)$ is an input transformation of $(\tilde{P}, \tilde{S})$ under $V$ if

$$
\left[\begin{array}{ll}
\tilde{P}_{11} & \tilde{P}_{12} \\
\tilde{P}_{21} & \tilde{P}_{22}
\end{array}\right]=\left[\begin{array}{ll}
P_{11} & P_{12} \\
P_{21} & P_{22}
\end{array}\right]\left[\begin{array}{cc}
I & 0 \\
0 & V
\end{array}\right] \quad \text { and } \quad S=V \tilde{S}
$$

If $V$ is wide and full normal rank it is an input reduction, and if strictly wide, it is a strict input reduction.

Definition 14. We say $(P, S)$ is input-minimal if the matrix $\left[\begin{array}{l}P_{12} \\ P_{22}\end{array}\right]$ is tall and has full normal rank.

Theorem 15. There always exists an input reduction to an input-minimal system.

Corollary 16. Consider a system $(P, S)$, and consider the set of all possible systems $\left(P^{\prime}, S^{\prime}\right)$ that can be obtained from it via an input transformation. This set contains a QI system if and only if an input-minimal transformation of $(P, S)$ is $Q I$.

As in the case of output reductions, Corollary 16 provides an additional sufficient condition for internal quadratic invariance. If we test the input-minimal system provided by Theorem 15, and the result is not QI, then no input transformation can yield a QI system.

\subsection{Joint Input-Output Reduction}

The ideas of output reduction and input reduction are independent. Specifically, the $U$ and $V$ from Theorems 12 and 15 can be constructed independently, and the reduction shown in Figure 3 will be both output-minimal and input-minimal.
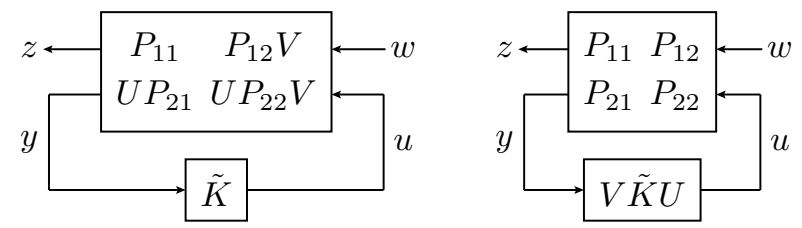

Figure 3: Equivalent interconnection obtained by a joint input-output transformation.

We can compute the reduced system directly from $U$ and $V$ using

$$
\begin{aligned}
& {\left[\begin{array}{ll}
P_{11} & P_{12} \\
P_{21} & P_{22}
\end{array}\right]=\left[\begin{array}{cc}
I & 0 \\
0 & U^{\dagger}
\end{array}\right]\left[\begin{array}{cc}
\tilde{P}_{11} & \tilde{P}_{12} \\
\tilde{P}_{21} & \tilde{P}_{22}
\end{array}\right]\left[\begin{array}{cc}
I & 0 \\
0 & V^{\dagger}
\end{array}\right]} \\
& \text { and } \quad S=V \tilde{S} U
\end{aligned}
$$

Definition 17. $(P, S)$ is minimal if it is both outputminimal and input-minimal. 
We can summarize the main result of this paper in the following sufficient condition for the internal quadratic invariance of a system. In order to check it, we only need to examine the minimal form obtained from Theorems 12 and 15.

Theorem 18. Consider $(\tilde{P}, \tilde{S})$, and consider the set of all $(P, S)$ that can be obtained from it via a combination of input and output transformations. This set contains a QI system if and only if a minimal transformation of $(\tilde{P}, \tilde{S})$ is $Q I$.

\section{Example Revisited}

An Example of Reduction. Consider once again the example from Section 3. We notice that it is not outputminimal because $\left[\begin{array}{ll}\tilde{P}_{21} & \tilde{P}_{22}\end{array}\right]$ doesn't have full normal rank, and so an output reduction is possible:

$$
\begin{aligned}
{\left[\begin{array}{ll}
\tilde{P}_{21} & \tilde{P}_{22}
\end{array}\right] } & =\left[\begin{array}{cccc}
\frac{1}{2} C_{1} & G_{1} & 0 & 0 \\
\frac{1}{3} C_{1} & \frac{2}{3} G_{1} & 0 & 0 \\
\frac{1}{5} C_{2} & \frac{2}{5} G_{2} & \frac{3}{5} G_{3} & G_{3}
\end{array}\right] \\
& =\underbrace{\left[\begin{array}{cc}
\frac{1}{2} I & 0 \\
\frac{1}{3} I & 0 \\
0 & \frac{1}{5} I
\end{array}\right]}_{U}\left[\begin{array}{cccc}
C_{1} & 2 G_{1} & 0 & 0 \\
C_{2} & 2 G_{2} & 3 G_{3} & 5 G_{3}
\end{array}\right] .
\end{aligned}
$$

Similarly, $\left[\begin{array}{c}\tilde{P}_{12} \\ \tilde{P}_{22}\end{array}\right]$ is not input-minimal, so an input reduction is possible as well:

$$
\begin{aligned}
{\left[\begin{array}{c}
\tilde{P}_{12} \\
\tilde{P}_{22}
\end{array}\right]=} & {\left[\begin{array}{ccc}
2 B_{1} & 3 B_{2} & 5 B_{2} \\
G_{1} & 0 & 0 \\
\frac{2}{3} G_{1} & 0 & 0 \\
\frac{2}{5} G_{2} & \frac{3}{5} G_{3} & G_{3}
\end{array}\right] } \\
= & {\left[\begin{array}{cc}
B_{1} & B_{2} \\
\frac{1}{2} G_{1} & 0 \\
\frac{1}{3} G_{1} & 0 \\
\frac{1}{5} G_{2} & \frac{1}{5} G_{3}
\end{array}\right] \underbrace{\left[\begin{array}{ccc}
2 I & 0 & 0 \\
0 & 3 I & 5 I
\end{array}\right]}_{V} . }
\end{aligned}
$$

We can compute the reduced system using $U$ and $V$ :

$$
\begin{aligned}
{\left[\begin{array}{ll}
P_{11} & P_{12} \\
P_{21} & P_{22}
\end{array}\right]=\left[\begin{array}{cc}
I & 0 \\
0 & U^{\dagger}
\end{array}\right]\left[\begin{array}{cc}
\tilde{P}_{11} & \tilde{P}_{12} \\
\tilde{P}_{21} & \tilde{P}_{22}
\end{array}\right]\left[\begin{array}{cc}
I & 0 \\
0 & V^{\dagger}
\end{array}\right] } \\
=\left[\begin{array}{ccc}
P_{11} & B_{1} & B_{2} \\
C_{1} & G_{1} & 0 \\
C_{2} & G_{2} & G_{3}
\end{array}\right] \\
S=V \tilde{S} U=\left\{V\left[\begin{array}{ccc}
K_{1} & 0 & 0 \\
0 & K_{2} & 0 \\
0 & 0 & K_{3}
\end{array}\right] U \mid K_{i} \in \mathcal{R}\right\} \\
=\left\{\left[\begin{array}{cc}
K_{1} & 0 \\
K_{2} & K_{3}
\end{array}\right] \mid K_{i} \in \mathcal{R}\right\} .
\end{aligned}
$$

This resulting system is QI, as shown in Section 3.
Networked System Example. In this section, we will show a more complicated example of an internally quadratically invariant system. Suppose we have two discrete-time systems $G_{1}$ and $G_{2}$, controlled by $K_{1}$ and $K_{2}$ respectively. Controller $K_{1}$ receives a measurement from $G_{1}$, and a one-timestep-delayed measurement from $G_{2}$. Similarly, $K_{2}$ receives a measurement from $G_{2}$, and a one-timestep-delayed measurement from $G_{1}$. Now further suppose that $G_{1}$ and $G_{2}$ are coupled, so that $G_{1}$ has an additional input that depends on the state of $G_{2}$, and vice-versa. The coupling has a delay of one timestep. See Figure 4.

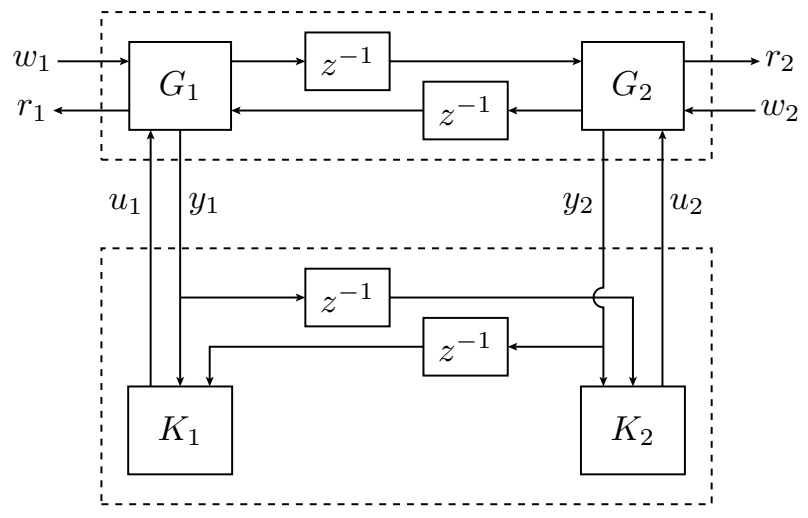

Figure 4: Two coupled systems with controllers that receive delayed measurements

Let $G_{1}$ be the stable second-order plant with discretetime state-space equations:

$$
\begin{aligned}
x_{1}(t+1) & =\left[\begin{array}{cc}
0.9 & 0.3 \\
-0.6 & 0.8
\end{array}\right] x_{1}(t)+\left[\begin{array}{l}
0 \\
1
\end{array}\right] u_{1}(t)+\left[\begin{array}{l}
0 \\
1
\end{array}\right] v_{2}(t-1) \\
r_{1}(t) & =\left[\begin{array}{ll}
1 & 0 \\
0 & 0
\end{array}\right] x_{1}(t)+\left[\begin{array}{l}
0 \\
\mu
\end{array}\right] u_{1}(t) \\
{\left[\begin{array}{l}
y_{1}(t) \\
v_{1}(t)
\end{array}\right] } & =\left[\begin{array}{cc}
1 & 0 \\
0.1 & 0.2
\end{array}\right] x_{1}(t)+\left[\begin{array}{l}
1 \\
0
\end{array}\right] w_{1}(t),
\end{aligned}
$$

where $r_{i}$ are the regulated outputs we wish to keep small, $u_{i}$ are the inputs provided by the controllers, and $v_{i}$ is the coupling between the two systems. The equations are the same for $G_{2}$, except the subscripts 1 and 2 are interchanged. Taking $z$-transforms and eliminating the state $x$, we obtain the plant

$$
\left[\begin{array}{l}
r \\
y
\end{array}\right]=\left[\begin{array}{ll}
P_{11} & P_{12} \\
P_{21} & P_{22}
\end{array}\right]\left[\begin{array}{l}
w \\
u
\end{array}\right]
$$

where the various transfer functions are:

$$
\begin{gathered}
P_{11}=0, \quad P_{21}=I, \quad P_{12}=\left[\begin{array}{c}
P_{22} \\
\mu I
\end{array}\right], \\
P_{22}=\frac{6 z}{\Delta}\left[\begin{array}{cc}
2 z\left(10 z^{2}-17 z+9\right) & 4 z-3 \\
4 z-3 & 2 z\left(10 z^{2}-17 z+9\right)
\end{array}\right],
\end{gathered}
$$

and $\Delta=\left(20 z^{3}-34 z^{2}+14 z+3\right)\left(20 z^{3}-34 z^{2}+22 z-3\right)$. The controller has a special structure, because of the delays 
associated with the measurements:

$$
S=\left\{\left[\begin{array}{cc}
K_{11} & z^{-1} K_{12} \\
z^{-1} K_{21} & K_{22}
\end{array}\right] \mid K_{i j} \in \mathcal{R}_{p}\right\} .
$$

It is straightforward to verify that $K P_{22} K \in S$ for all $K \in S$. So we conclude that $(P, S)$ is QI. A more general version of this problem is analyzed in [9].

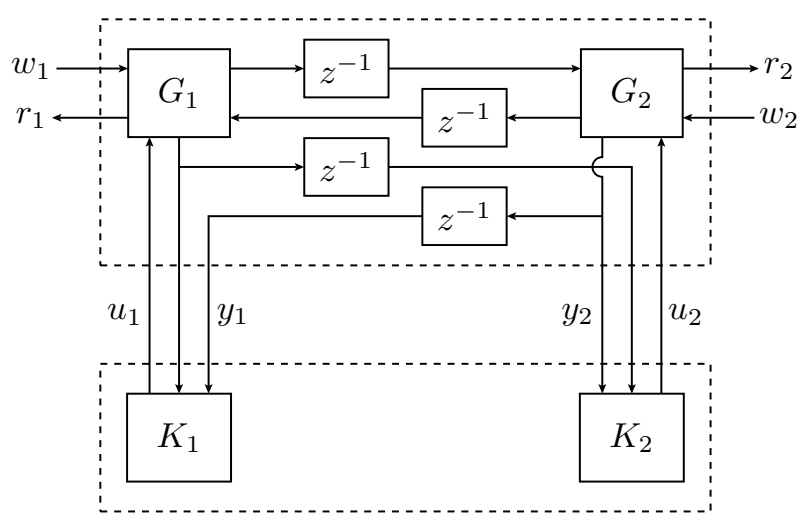

Figure 5: Alternate diagram for the system of Figure 4

Note that there are multiple ways of generating a pair $(P, S)$ that represents the system in Figure 4. For example, we could absorb the measurement delays into $P$, as shown in Figure 5 , so that $\tilde{P}_{11}=P_{11}, P_{12}=P_{12}$,

$$
\begin{gathered}
\tilde{P}_{21}=\left[\begin{array}{cc}
1 & 0 \\
z^{-1} & 0 \\
0 & 1 \\
0 & z^{-1}
\end{array}\right] \text {, and } \\
\tilde{P}_{22}=\frac{6 z}{\Delta}\left[\begin{array}{cc}
2 z\left(10 z^{2}-17 z+9\right) & 4 z-3 \\
2\left(10 z^{2}-17 z+9\right) & (4 z-3) / z \\
4 z-3 & 2 z\left(10 z^{2}-17 z+9\right) \\
(4 z-3) / z & 2\left(10 z^{2}-17 z+9\right)
\end{array}\right] .
\end{gathered}
$$

The new information constraint is sparse

$$
\tilde{S}=\left\{\left[\begin{array}{cccc}
K_{1} & 0 & 0 & K_{2} \\
0 & K_{3} & K_{4} & 0
\end{array}\right] \mid K_{i} \in \mathcal{R}_{p}\right\} .
$$

It is straightforward to verify that $\tilde{K} \tilde{P}_{22} \tilde{K}$ does not have the same sparsity pattern as $\tilde{S}$; it is a full matrix in general. Therefore, $(\tilde{P}, \tilde{S})$ is not QI. Had we used this representation for our problem, we would still be able to transform it into a QI representation. Check for minimality; nrank $\left[\begin{array}{cc}\tilde{P}_{21} & \tilde{P}_{22}\end{array}\right]=2$, which is not full normal rank, so our system is not output-minimal. Then use Theorem 12, to factor

$$
\left[\begin{array}{ll}
\tilde{P}_{21} & \tilde{P}_{22}
\end{array}\right]=\left[\begin{array}{cc}
1 & 0 \\
z^{-1} & 0 \\
0 & 1 \\
0 & z^{-1}
\end{array}\right]\left[\begin{array}{ll}
P_{21} & P_{22}
\end{array}\right]
$$

We can also verify that $S=\tilde{S} U$, which shows that this output transformation actually recovers $(P, S)$, our first representation of the system. Therefore, $(\tilde{P}, \tilde{S})$ is internally QI.

\section{Conclusion}

Quadratic invariance is a property of the actuatormeasurement transfer function $P_{22}$ and the information constraint set $S$. We showed in this paper that by including conditions on $P_{12}$ and $P_{21}$ as well, we can characterize a class of problems that can be reduced to QI problems, even though they are not QI themselves.

This work broadens the QI class of decentralized control problems, and is a step towards a more complete characterization of tractable problems in decentralized control.

\section{References}

[1] R. Bansal and T. Basar. Stochastic teams with nonclassical information revisited: When is an affine law optimal? IEEE Transactions on Automatic Control, 32(6):554-559, 1987.

[2] V. D. Blondel and J. N. Tsitsiklis. A survey of computational complexity results in systems and control. Automatica, 36(9):1249-1274, 2000.

[3] S. Boyd and C. Barratt. Linear controller design: limits of performance. Prentice-Hall, 1991.

[4] Y-C. Ho and K-C. Chu. Team decision theory and information structures in optimal control problems-Part I. IEEE Transactions on Automatic Control, 17(1):15-22, 1972.

[5] T. Kailath. Linear systems. Prentice-Hall Englewood Cliffs, NJ, 1980.

[6] R. Radner. Team decision problems. The Annals of Mathematical Statistics, 33(3):857-881, 1962.

[7] M. Rotkowitz. On information structures, convexity, and linear optimality. In IEEE Conference on Decision and Control, pages 1642-1647, 2008.

[8] M. Rotkowitz and S. Lall. Decentralized control information structures preserved under feedback. In IEEE Conference on Decision and Control, volume 1, pages 569-575, 2002.

[9] M. Rotkowitz and S. Lall. A characterization of convex problems in decentralized control. IEEE Transactions on Automatic Control, 51(2):274-286, 2006.

[10] H. S. Witsenhausen. A counterexample in stochastic optimum control. SIAM Journal on Control, 6:131, 1968. 